\title{
Laser Mode-Locking with Saturable Absorbers
}

\author{
ELSA M. GARMIRE AND AMNON YARIV, MEMBER, IEEE
}

\begin{abstract}
In this paper we describe a mode-locked laser in terms of traveling pulses of light. We show that the energy absorbed by a saturable absorber is a minimum if the pulselength is a minimum, and that two pulses are essentially as favorable as one if they meet at the position of the dye cell. Under steady-state pulsing conditions, however, we show that the pulses will have a width which depends on their energy. We find that for parameters appropriate to present $\mathrm{Nd}$ : glass experiments, the expected length is about $10^{-11}$ seconds, in agreement with observations. Finally, we demonstrate the rather surprising result that a linearly dispersive medium does not broaden the mode-locked laser pulses to first order.
\end{abstract}

\section{INTRODUCTION}

T THE GENERATION of ultrashort optical pulses by means of mode-locking in multimode lasers is a problem of considerable current interest. In the first experimental demonstration of this phenomenon, ${ }^{[1]}$ the mode-locking was obtained by means of loss modulation applied at a frequency equal to that of the longitudinal mode spacing $c / 2 L$, where $L$ is the length of the optical cavity. Theoretical analyses of this effect ${ }^{[2]-[4]}$ show that the duration of the pulses emitted by mode-locked lasers is not limited by the decay time constant of the optical resonator. The pulses are due, instead, to interference between the oscillating laser modes, which causes the pulse duration to have a value of $\sim \Delta \nu^{-1}$, where $\Delta \nu$ is the natural linewidth of the amplifying transition.

More recently, it has been shown experimentally ${ }^{[5]-[6]}$ that mode-locking may result from the mere insertion of a saturable absorber within the optical resonator of $Q$-switched lasers. In the latter reference, mode-locked pulses were obtained at $1.06 \mu$ from glass: $\mathrm{Nd}^{3+}$ with $100 \AA$ bandwidth so that the theoretical minimum pulsewidth is $3.7 \times 10^{-13}$ seconds. More recent experiments show that the pulsewidth is ten times the minimum width $^{[7],[8]}$ and increases as the energy of the pulse increases. ${ }^{[s]}$

In this paper we present a theoretical discussion of the characteristics of lasers mode-locked by means of a saturable absorber located at an arbitrary point inside the optical resonator. A rigorous theoretical solution for the mode-locked glass: $\mathrm{Nd}^{3+}$ laser would involve an exact calculation for the behavior of thousands of modes. Since this is not feasible, we use approximate methods to study certain aspects of the problem. The cavity modes are expressed as traveling pulses of light rather than as standing waves, as in previous analyses. We then examine the behavior of the saturable absorber when it is subjected to a light pulse of given energy and width. This

Manuscript received February 13, 1967. The work reported in this paper was supported by the USAF Avionics Laboratory through Contract AF33(615)-67C-1055.

The authors are with the Dept. of Elec. Engrg., California Institute of Technology, Pasadena, Calif. analysis allows us to discuss a laser whose output consists of pulses which are longer than the theoretical minimum pulsewidth.

We shall demonstrate that the energy absorbed by the saturable absorber from a traveling light pulse of fixed energy is a minimum if the pulselength is a minimum. As long as the laser oscillates in such a way as to minimize the losses in the absorber, its output will consist of a family of light pulses of length $c / \Delta \nu$ separated by $2 L$, and the laser will be fully mode-locked. In this type of oscillation, a single pulse travels back and forth inside the resonator.

We shall further show that when the absorber is placed a distance $d$ from the end of the optical resonator, a type of oscillation in which two traveling pulses are present at any one time inside the cavity is almost as favorable as that of the single pulse described above. In this case, the two pulses meet at the absorber, so that the output consists of a biperiodic pulse train with pulses separated by a distance $2 d$.

Experiments have shown that a steady-state condition is reached in which successive pulses in the laser output have roughly the same energy and width. In this case, the pulsewidths are much larger than the minimum theoretical pulsewidth and appear to increase as the energy increases. We show theoretically that the requirements of steady-state pulse generation result in a pulselength which depends on the pulse energy. We find that for values of parameters corresponding to present experiments, the pulselength should increase somewhat as the energy increases and is the order of magnitude of $10^{-11}$ seconds. We also demonstrate the rather surprising result that dispersion does not broaden the ultrashort pulses to first order.

\section{Description of Laser Modes}

Let us show that the radiation distribution inside a mode-locked laser cavity consists of a light pulse of width $L / n$ bouncing back and forth between the reflectors at the velocity of light. Consider a TEM-like optical resonator of length $L$ supporting optical modes of the type

$$
E_{a}(x, t)=E_{a} \sin \left[(m+a) \frac{\pi x}{L}\right] \sin \left[\left(\omega_{0}+a \omega\right) t+\phi_{a}\right] .
$$

Since $\omega_{0}=m \pi c / L$ and $\omega=\pi c / L$, trigonometric manipulation allows us to write the standing waves in the form of traveling waves. Summing over all $n$ modes yields:

$$
\begin{gathered}
E(x, t)=\frac{1}{2} \sum_{a=-n / 2}^{n / 2} E_{a}\left\{\cos \left[(m+a) \frac{\pi}{L}(x-c t)-\phi_{a}\right]\right. \\
\left.-\cos \left[(m+a) \frac{\pi}{L}(x+c t)+\phi_{a}\right]\right\} .
\end{gathered}
$$


We have $n \simeq(L / c) \Delta \nu$, where $\Delta \nu$ is the amplification bandwidth of the laser.

When a laser is mode-locked, all phases $\phi_{a}$ are equal. This has been shown previously in the case of externally modulated lasers ${ }^{[2]-\{4]}$ and in the case of a distributed nonlinear gain ${ }^{[4]}$. It will be shown below that a saturable absorber also has this property. If the phases $\phi_{a}$ are all constant (or vary by an amount which is proportional to $a$ ), they may be taken as zero by a shift of the time reference. In that case, the field inside a mode-locked laser may be written as:

$$
\begin{aligned}
E(x, t)= & {\left[\sum_{-n / 2}^{n / 2} E_{a} e^{(i a \pi / L)(x-c t)}\right] \cos \frac{m \pi}{L}(x-c t) } \\
& +\left[\sum_{-n / 2}^{n / 2} E_{a} e^{(i a \pi / L)(x+c t)}\right] \cos \frac{m \pi}{L}(x+c t) .
\end{aligned}
$$

By extending the values of $x$ outside the boundaries $0 \leq x \leq L$ of the optical resonator, the radiation distribution is seen to consist of a family of pulses of width (in space) $\sim L / n$ spaced by $2 L$ which are traveling to the right at the velocity of light. A second such family is traveling to the left. Inside the optical resonator, the effect is that of a single pulse of width $L / n=c / \Delta \nu$ bouncing back and forth between the reflectors at the velocity of light $c$.

An exact description of mode-locking by means of saturable absorbers would necessitate calculation of the effect of the dye on the phase and amplitude of each mode. In the presence of only a few modes, the character of the output radiation ${ }^{[9]}$ and the absorption properties of the dye $^{[10]}$ for each mode have been calculated. Neither of these methods is easily applicable to determining the characteristics of the output radiation when many modes couple to form short pulses of light. For this reason, we propose to approximate the radiation of a mode-locked system by pulses of light and to study their behavior in space and time rather than calculate the amplitude and phase of individual modes. We will consider the radiation distribution inside the optical resonator as a pulse of energy $E$ and width $L_{p}$ bouncing back and forth. If $L_{p}=L / n$, then all phases $\phi_{a}$ are equal and the cavity is fully mode-locked. The analysis ignores the issue of how the pulses are formed. It assumes that they exist and, furthermore, that their width $L_{p}$ is small compared to the resonator length $L$. We then proceed to find the conditions leading to minimum absorption.

\section{EFFect of Absorber}

We will show that the energy absorbed by the saturable absorber from a pulse of light is a minimum consistent with the amplification bandwidth. This has the effect of locking all phases $\phi_{a}$ together, the condition which gives a minimum pulsewidth for the radiation distribution [eq. (2)]. Our formulation will consider the behavior of the dye in space and time and assume that it behaves in the same manner for all the frequencies (modes) within the effective bandwidth. This assumption is necessary to make the calculation tractable. In this case we can treat the absorption of the dye using rate equations.

It has been shown that the Kodak 9740 dye used as a saturable absorber with $\mathrm{Nd}$ lasers can be described with the aid of a simple two-level model with an effective lifetime of the upper state of $2 \times 10^{-11}$ seconds. ${ }^{[11]} \mathrm{We}$ assume that the dye fully recovers in a transit time of the laser pulse. This will be true as long as the dyes used are not affected by longer-lived states. Slow recovery has not been observed in the Kodak dye. ${ }^{[11]}$

We write rate equations for the energy levels of the dye and the intensity of the light pulse. Taking the population densities of the upper and lower states as $\mathrm{N}_{2}$ and $N_{1}$, respectively, and letting $I$ represent the energy flux of the pulse, we can write

$$
\frac{d N_{1}}{d t}=R\left(N_{2}-N_{1}\right)+S N_{2} ; \quad N_{a}=N_{1}+N_{2}
$$

and

$$
\frac{d}{d x}\left(\frac{I}{\hbar \omega}\right)=-R\left(N_{1}-N_{2}\right)
$$

where $S$ is the spontaneous emission rate, $R$ is the rate of stimulated emission, and $N_{a}$ is the total density of dye molecules.

If at $t=0, N_{1}=N_{a},(4)$ and (5) can be solved to give the light intensity absorbed per unit length as a function of time:

$$
-\frac{d}{d x}\left(\frac{I}{\hbar \omega}\right)=\frac{N_{a} R}{2 R+S}\left[S+2 R e^{-(2 R+S) t}\right]
$$

Since $R$ is proportional to the field intensity, $R$, in general, is a function of $x$, distance through the dye cell, and of time.

In order to simplify calculations, we will assume that the light intensity is a constant for a time $T=L_{p} / c$ and zero for all other times. The light pulse consists of $E$ photons $/ \mathrm{cm}^{2}$ evenly spread out over the time $T$. We shall further assume that the light intensity is not greatly depleted as a function of distance through the dye cell. This is true for sufficiently small total absorption and holds rather well for the experimental absorptions of about 30 percent unsaturated loss per pass. These assumptions allow a simple integration of (6) with respect to $x$ and $t$. The total number of photons $/ \mathrm{cm}^{2} E_{a}$ absorbed by a dye cell of thickness $L_{a}$ in a single traversal of the light pulse is then

$$
E_{a}=\frac{N_{a} \sigma_{a} E S T L_{a}}{2 \sigma_{a} E+S T}+\frac{2 N_{a}\left(\sigma_{a} E\right)^{2} L_{a}}{\left(2 \sigma_{a} E+S T\right)^{2}}\left(1-e^{-\left(2 \sigma_{a} E+S T\right)}\right) .
$$

We have made use here of the fact that $R T=\sigma_{a} E$, where $\sigma_{a}$ is the absorption cross section per dye molecule.

The absorption of the dye is a monotonically decreasing function of the pulselength. This can be seen by observing that there is no positive value of $T$ which satisfies $\partial E_{a} / \partial T=0$. It can be shown that the dependence of $E_{a}$ on pulselength always comes in through the quantity 
$2 \sigma_{a} E+S T$. Thus the absorption of the dye ceases to be a strong function of pulselength if $S T \ll 2 \sigma_{a} E$; that is, if the rate of spontaneous emission is much less than the rate of stimulated emission. The absorption of a pulse lasting the order of the upper state lifetime is not much different from the absorption of pulses of the same energy which are much shorter. This suggests that dyes with much shorter lifetimes may be necessary to produce $10^{-13}$ ' second pulses in a $\mathrm{Nd}:$ glass laser.

We have shown that the loss in a saturable absorber is a minimum if the pulselength is a minimum. However, if the pulse becomes so short that it contains spectral components which lie outside the amplification bandwidth of the laser rod, these components will not grow. Thus the action of the saturable absorber will tend to stabilize at a pulselength about $c / \Delta \nu$, where $\Delta \nu$ is the laser linewidth. We have seen that such a pulselength corresponds to the case where all the phases $\phi_{a}$ are equal, so we have shown that a saturable dye locks the modes of a laser, and the radiation consists of a family of pulses of length $c / \Delta \nu$ separated by $2 L$.

The previous analysis assumed a homogeneously broadened line for the saturable absorber. The pulse-shortening effect of the dye produces sidebands which transfer energy of a fixed phase throughout the whole laser line, independent of the homogeneous or inhomogeneous linewidth of the dye. Thus we expect that the presence of inhomogeneous broadening should not greatly alter the mode-locking properties of the saturable dye.

It may be of interest to point out that in our analysis and in the relevant experiments, we assume all the modes have nearly equal gain. In this case, the saturable dye locks all the modes together. This is to be contrasted with the tendency of a saturable dye to concentrate energy in a single mode if this mode has a higher $Q$ than any of the other modes. This is the case where a combination of a saturable dye and passive mode discrimination leads to single mode operation. ${ }^{[12]}$

\section{Effect of Dye Posttion}

If the dye cell is placed at an arbitrary point within the cavity, one must consider the possibility that instead of only one light pulse within the cavity at any one time, there now can be two which meet at the dye cell, forming a standing wave of higher amplitude and, consequently, smaller relative absorption. In each complete period, however, each light pulse must go through the dye cell once by itself, resulting in additional absorption. We will show that to first order the energy loss in the dye cell is the same whether the light energy $E$ consists of one or two pulses. To second order, however, the single pulse is slightly favored.

Let us consider cases in which $\sigma_{a} E$ and $S T<1$, so the exponential in (7) can be expanded in a Taylor series. The number of photons absorbed from a single pulse of $E$ photons per $\mathrm{cm}^{2}$ and time $T$ in a complete round trip through the cavity (two passes through the cell) is

$$
\begin{aligned}
E_{a 1}=2 N_{a} L_{a} \sigma_{a} E[1- & \sigma_{a} E\left(1-\frac{S T}{3}\right) \\
& \left.+\frac{2}{3}\left(\sigma_{a} E\right)^{2}(1-S T)+\cdots\right] .
\end{aligned}
$$

When two pulses each of energy $E / 2$ cross each other in the dye cell, they combine to form a standing wave so that the photon density in the cell becomes $2 E / L_{p} \sin ^{2} k x$ instead of $E / L_{p}$. The number of absorbed photons $/ \mathrm{cm}^{2}$ $E_{a}$ for this crossing must be spatially averaged. The total energy absorbed in a complete round trip from the two pulses must also include two single passes in which $E$ is replaced by $E / 2$. This results in a total energy absorption of

$$
\begin{aligned}
E_{a 2}=2 N_{a} L_{a} \sigma_{a} E[1 & -\sigma_{a} E\left(1-\frac{S T}{3}\right) \\
& \left.+\frac{11}{12}\left(\sigma_{a} E\right)^{2}(1-S T)+\cdots\right] .
\end{aligned}
$$

Comparison of (8) and (9) indicates that to first order the dye absorbs the same amount whether all light energy is concentrated into one pulse or is divided into two. To second order, a single pulse is slightly favored. When two families of pulses occur, it is clear from this analysis that they will be separated in time by $2 d / c$ where $d$ is the distance of the dye cell to the end of the cavity. These results are in agreement with observations of deMaria et al. ${ }^{[13]}$ who find that when the dye cell is placed away from the end of the cavity it is usually somewhat more difficult to obtain two families of pulses than to obtain one.

When the dye cell is placed in the center of the cavity, the two pulses never have to go through the dye cell alone and

$$
E_{a 2}=2 N_{a} L_{a} \sigma_{a} E\left[1-\sigma_{a} E \frac{3}{2}\left(1-\frac{S T}{3}\right)+\cdots\right] .
$$

This is less than $E_{a 1}$ as given by (8) so that pulses separated by $L / c$ rather than $2 L / c$ are clearly preferred. This agrees with experiments and with the theoretical results of Statz et al. ${ }^{19 !}$ If the dye cell is considerably thicker than the length of the light pulses, however, the benefits of the high amplitude standing waves will be reduced by the fact that each pulse must travel part of the way through the cell by itself. In very thick cells single pulse operation, i.e., pulse separation of $2 L / c$, may result.

\section{Determination of Pulsewidth}

It has been shown that the energy loss in the absorber is a minimum if the pulselength is a minimum, but that the absorber becomes relatively ineffective in selecting pulses shorter than the lifetime of its upper state. To determine the steady-state pulselength, it is necessary to consider the conditions of steady-state oscillation of the laser as a whole. In this case, the energy loss in the dye 
plus the linear cavity losses must equal the energy gain in the laser rod. Since the dye losses depend on pulselength, this condition determines the steady-state pulselength as a function of the energy per pulse.

The amplification of the glass : $\mathrm{Nd}^{3+}$ laser rod will not depend on pulselength since the shortest relaxation time in the active levels is very much longer than the duration of the ultrashort pulses. Thus the laser rod responds only to the total energy in the light pulse. If the gain is unsaturated, the number of photons added to a pulse of $E$ photons by a rod of length $L_{r}$ is $L_{r} N_{r} \sigma_{r} E$, where $N_{r}$ is the initial inversion density of the Nd ions and $\sigma_{r}$ is the cross section per ion. $\sigma_{r} E \sim 10^{-3}$ in the cases of interest. Using (8) for the absorber loss, $\beta E$ for the cavity losses per pass, and the above $\mathrm{Nd}$ gain, the steady-state condition yields the following equation for the pulselength as a function of energy:

$$
\frac{S T}{3}=1-\frac{L_{a} N_{a} \sigma_{a}+\beta-L_{r} N_{r} \sigma_{r}}{\sigma_{a} E} .
$$

The energy-dependent term has the form of

$$
\frac{\text { linear loss - linear gain }}{\text { degree of dye saturation }} \text {. }
$$

The degree of dye saturation $\sigma_{a} E$ is roughly the number of photons absorbed by the dye per pass divided by the number of dye molecules or $E_{a 1} / 2 N_{a} L_{a}$ [see (8)]. In typical cases, this is about 0.3 . For (linear gain-linear loss) of about $0.2, S T \backsim 1$. In the Eastman 9740 reversible dye solution used with $\mathrm{Nd}$, the spontaneous decay time is $2 \times 10^{-11}$ seconds, ${ }^{[11]}$ so that typical steady-state pulselengths will be the order of $10^{-11}$ seconds. It is interesting to note that the pulselength broadens with increasing energy. Conversely it narrows as the energy decreases, in agreement with experimental observations. ${ }^{183}$

\section{EFfect of Dispersion}

It might be thought that the large pulsewidths observed experimentally are due to dispersion of the refractive index in the laser rod. We shall show that a linearly dispersive medium does not lengthen the individual pulses of a repetitive pulse train traversing it, to first order. The second-order effect broadens the pulselength only a part in $10^{-4}$.

While our previous analysis has not discussed the phases of specific modes under conditions other than full mode-locking, it is very necessary in this case to study the behavior of individual modes. Consider a family of pulses similar to those expressed in (2), traversing a medium whose refractive index is a function of frequency. It is not necessary that the pulses be fully mode-locked. Since each of the frequency components of the pulse are equally spaced, the refractive index of the ath component can be written as

$$
n=n_{0}+a \frac{\partial n}{\partial \omega} \frac{\pi c}{L}+\frac{1}{2} a^{2}\left(\frac{\pi c}{L}\right)^{2} \frac{\partial^{2} n}{\partial \omega^{2}}+\cdots
$$

In that case, a pulse train can be written as

$$
\begin{aligned}
E= & \cos \frac{m n_{0} \pi}{L}(x-c t) \\
& \cdot \sum_{-N / 2}^{N / 2} E_{a} \cos \left[\frac{a \pi}{L}\left(n^{\prime} x-c t\right)+a^{2} \frac{\pi}{L} \epsilon x+\phi_{a}\right] \\
- & \sin \frac{m n_{0} \pi}{L}(x-c t) \\
& \cdot \sum_{-N / 2}^{N / 2} E_{a} \sin \left[\frac{a \pi}{L}\left(n^{\prime} x-c t\right)+a^{2} \frac{\pi}{L} \epsilon x+\phi_{a}\right],
\end{aligned}
$$

where

$$
n^{\prime}=n_{0}+\omega_{0} \frac{\partial n}{\partial \omega}
$$

and

$$
\epsilon=\frac{\pi c}{L}\left[\frac{\partial n}{\partial \omega}+\frac{\omega_{0}}{2} \frac{\partial^{2} n}{\partial \omega^{2}}\right] .
$$

From (12), it is clear that to first order in $a$, the linear dispersion merely increases the effective refractive index. To second order, however, the effect of the term containing $\epsilon$ is to broaden the pulse slightly. This broadening will be a part in $10^{-4}$, which is the order of magnitude of $N \epsilon$ in the glass : Nd, where $N$ is the number of modes comprising the pulses.

In the previous analysis, we assumed a pulse train of fixed frequency components was incident upon a dispersive medium. The analysis could have been made assuming the resonant frequencies of the laser cavity are altered by the dispersion. The results of such a calculation give the same small amount of pulse broadening. In any event, once the pulselength is much shorter than the cavity length, it is not clear that the cavity resonance conditions are important, since there is little standingwave character in the radiation. In this ease the mirrors merely fold the optical path of the traveling pulse.

\section{Conclusions}

The behavior of a laser mode-locked with a saturable absorber has been analyzed under certain conditions. We have shown that a convenient description of the cavity modes is traveling pulses of light. We demonstrated that the nonlinear absorption of a saturable dye tends to lock the modes of a laser resulting in pulses of minimum width $\Delta \nu^{-1}$. However, absorber loss does not depend strongly on the pulselength when the pulses are much shorter than the spontaneous lifetime of the dye. Since this time is $10^{-11}$ seconds in the Kodak dye, it may be necessary to find dyes with sherter lifetimes to produce a single train of $10^{-13}$ seconds pulses from $\mathrm{Nd}$.

We have shown that if the saturable absorber is localized at a distance $d$ from the end of the cavity, a biperiodic pulse train may occur with pulses separated by a distance $2 d$.

Under steady-state conditions, we have shown that the pulses have a width that depends on their energy. For 
values of parameters corresponding to present experiments, we found that the pulselength should increase somewhat as the energy increases and is the order of magnitude of $10^{-11}$ seconds, in agreement with observations.

Finally, we have shown that dispersion of the refractive index in the laser rod does not broaden the mode-locked pulses to first order.

\section{References}

[1] L. E. Hargrove, R. L. Fork, and M. A. Pollack, "Locking of $\mathrm{He}-\mathrm{Ne}$ laser modes induced by synchronous intracavity modulation," Appl. Phys. Lett., vol. 5, p. 4, 1964.

[2] M. DiDomenico, Jr., "Small-signal analysis of internal (Coupling-type) modulation of lasers," J. Appl. Phys., vol. 35, pp. 28702876, October 1964 .

[3] A. Yariv, "Internal modulation in multimode laser oscillators," J. Appl. Phys., vol. 36, pp. 388-391, February 1965.
[4] M. H. Crowell, "Characteristics of mode-coupled lasers," IEEE J. Quantum Electronics, vol. QE-1, pp. 12-20, April 1965. ${ }^{[5]} \mathrm{H}$. Mocker and R. J. Collins, "Mode competition and selflocking effects in a Q-switched ruby laser," A ppl. Phys. Lett., vol. 7, pp. $270-273$, November 15,1965 .

[6] A. J. DeMaria, D. A. Stetser, and H. Heynau, "Self modelocking of lasers with saturable absorbers," A ppl. Phys. Lett., vol. 8, pp. 174-176, April 1, 1966.

[7] J. A. Armstrong, "Measurement of picosecond laser pulse widths," Appl. Phys. Lett. vol. 10, pp. 16-18, January 1, 1967.

[8] M. J. Brienza and W. H. Glenn, Jr., post-deadline paper, Am. Phys. Soc., December 28, 1966, Stanford, Calif.

[9] H. Statz, G. A. deMars, and C. L. Tang, "Self locking of modes in lasers," (to be published).

[10] S. E. Schwarz and T. Y. Tan, "Wave interactions in saturable absorbers," Appl. Phys. Lett., vol. 10, pp. 4-7, January 1, 1967.

[11] B. Soffer, Korad Corporation, private communication.

[12] W. R. Sooy, "The natural selection of modes in a passive $Q$-switched laser," A ppl. Phys. Lett., vol. 7, pp. 36-37, July 15, 1965.

[13! A. J. DeMaria, D. A. Stetser, and W. H. Glenn, Jr., "Ultrashort light pulses," AAAS (to be published) and private communication.

\section{Correction}

Teiji Uchida, author of the paper, "Dynamic Behavior of Gas Lasers," which appeared on pages 7-16 of the January, 1967, issue of this Journal, has called the following to the attention of the Editor.

On page 9 , only the left-hand side of (36) should be multiplied by 2 as follows: $2 \cdot \Delta b$. 